The Astrophysicat Journal, 185:L37-L40, 1973 October 1

(c) 1973. The American Astronomical Society. All rights reserved. Printed in U.S.A.

\title{
INFRARED OBSERVATIONS OF TWO SYMMETRIC NEBULAE
}

\author{
Martin Cohen and Michael J. Barlow \\ Berkeley Astronomy Department, University of California \\ Received 1973 July 19
}

\begin{abstract}
Infrared observations are presented of NGC 7635, IC 1470, and their early-type central stars. Large excess fluxes are found at both 10 and $20 \mu$, which are interpreted as due to thermal emission by cool dust.

Subject headings: early-type stars - infrared sources
\end{abstract}

\section{INTRODUCTION}

NGC 7635 and IC 1470 are both members of a group of objects classified as "symmetric nebulae" by Johnson (1955). During an infrared photometric investigation of the central stars of several of these nebulae, a large excess of emission was found between 10 and 18 microns from BD $+60^{\circ} 2522$, in NGC 7635. Subsequently, during an independent examination of the AFCRL infrared survey (Price and Walker 1973), one of the unidentified sources in the catalog was recognized to be NGC 7635. Additionally we noticed that one of the identified sources was IC 1470. Infrared observations were then made of the central star of this object also.

\section{THE OBSERVATIONS}

A multifilter photometer was used on the University of Minnesota-University of California, San Diego, 60-inch $(152-\mathrm{cm})$ telescope on Mount Lemmon. The normal technique of sky-chopping was employed with $11^{\prime \prime}$ beams and a $15^{\prime \prime}$ throw. The detector was a liquid-helium bolometer operating near $1^{\circ} \mathrm{K} . \mathrm{BD}+60^{\circ} 2522$ was observed on 1972 December 22, and the 3.65- and 4.8-micron measurements were repeated on 1973 January 25 and June 16. IC 1470 was observed on 1972 December 23. The magnitudes and photometric errors (one standard deviation of the mean) of the two stars are included in tables 1 and 2, as are the available data from the AFCRL catalog (taken with a $10^{\prime} \times 3^{\prime}$ aperture). $U B V$ data are from Hiltner (1956) for $\mathrm{BD}+60^{\circ} 2522$ and from Kostjakova et al. (1968) for the central star of IC 1470. The 1.65-micron magnitude of $\mathrm{BD}+60^{\circ} 2522$ is from Allen (1973). Figure 1 presents the energy distributions of the two objects between 0.36 and 18 microns.

If the large fluxes seen by the rocket were uniformly distributed across the nebulae, then ground-based measurements with our sky-chopping system would discriminate against our detecting such smoothly varying sources. Consequently our detection of long-wavelength radiation implies a concentration of emission around the stars.

Color temperatures were derived using the longest wavelength fluxes, with the calibration in Cohen (1973). The notation $T_{c}\left(\lambda_{1}-\lambda_{2}\right)$ in tables 1 and 2 denotes the temperature of the blackbody, the ratio of whose fluxes at wavelengths $\lambda_{1}$ and $\lambda_{2}$ microns matches the observed ratio.

\section{DISCUSSION}

a) $N G C 7635$

A Hale Observatories photograph of NGC 7635 can be found in the Griffith Observer (ed. Roques 1973). This clearly shows a ring of nebulosity, which is NGC 


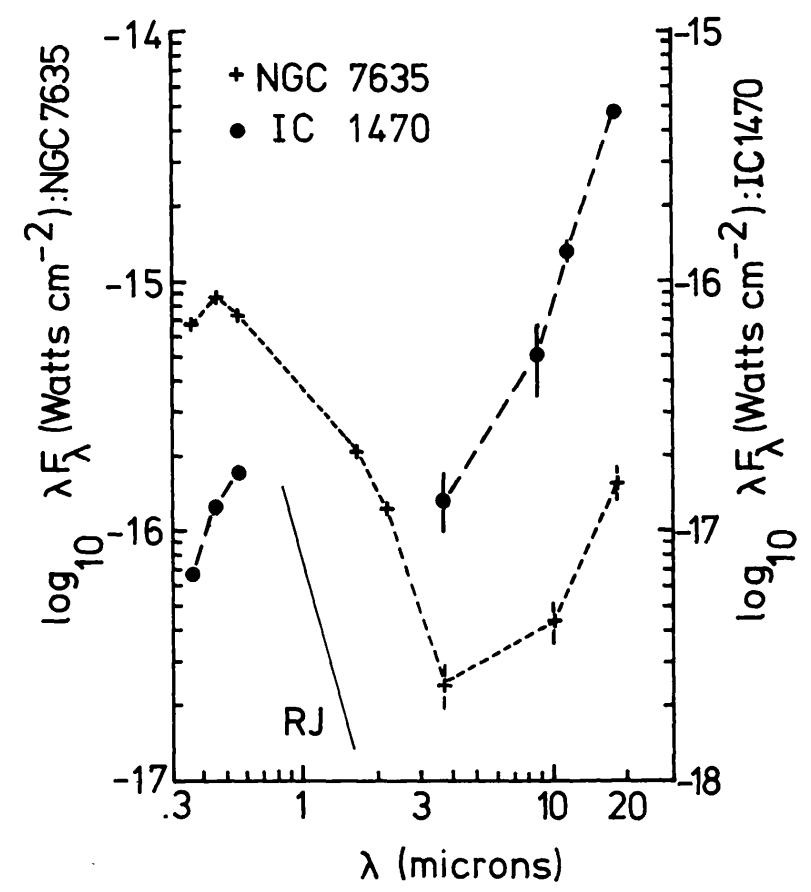

FIG. 1.-Energy distributions of NGC 7635 (left-hand ordinate scale) and IC 1470 (right-hand scale), from ground-based observations. RJ denotes the slope of the Rayleigh-Jeans tail of a blackbody.

TABLE 1

MAGNitudes AND LUMTNOSITIES FOR NGC $7635 / \mathrm{BD}+60^{\circ} 2522$

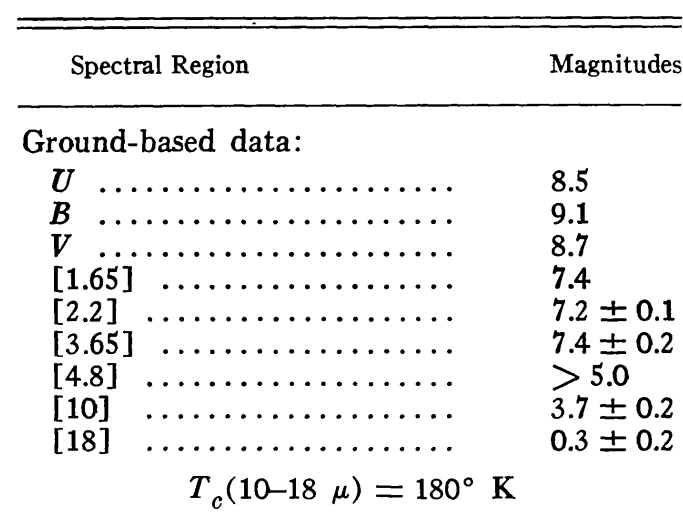

Rocket data:

$$
\begin{aligned}
& \text { [11] ................ None }
\end{aligned}
$$

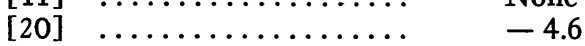

$$
\begin{aligned}
& T_{c}(11-20 \mu): \text { None }
\end{aligned}
$$

Note. $-L_{\text {UV }}($ radio $)=2.8 \times 10^{38}$ ergs $\mathrm{s}^{-1}$ $=7.3 \times 10^{4} L_{\odot} ;$ distance $=3.5 \mathrm{kpc} ; L_{\mathrm{IR}}\left(180^{\circ}\right.$ $\mathrm{K}$ blackbody, ground) $=2.9 \times 10^{36} \mathrm{ergs} \mathrm{s}^{-1}$ $=750 L_{\odot} ; L_{\mathrm{IR}}\left(180^{\circ} \mathrm{K}\right.$ blackbody, rocket $)=$ $2.2 \times 10^{38} \mathrm{ergs} \mathrm{s}^{-1}=5.7 \times 10^{4} L_{\odot}$.
TABLE 2

Magnitudes AND Luminositues FOR IC 1470

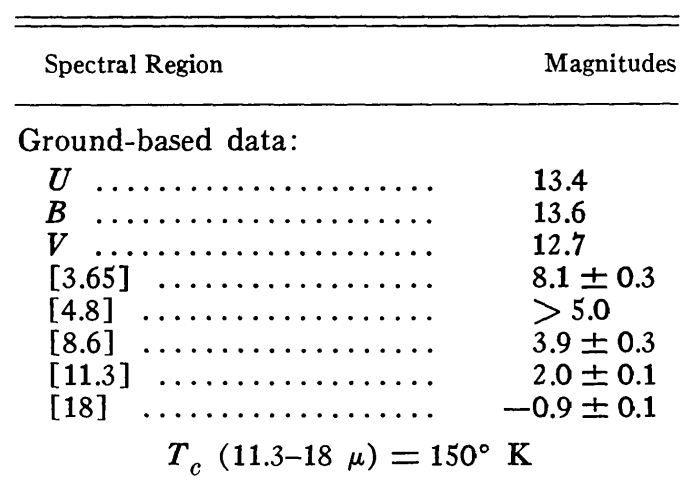

Rocket data:

$$
\begin{aligned}
& \text { [11] } \ldots \ldots \ldots \ldots \ldots \ldots \ldots \quad-1.7 \\
& {[20] \quad \ldots \ldots \ldots \ldots \cdots \cdots \cdots \cdots \cdots \cdots} \\
& T_{c}(11-20 \mu)=155^{\circ} \mathrm{K}
\end{aligned}
$$

Note. $-L_{\text {UV }}($ radio $)=1.4 \times 10^{38}$ ergs $\mathrm{s}^{-1}$ $=3.6 \times 10^{4} L_{\odot} ;$ distance $=4.3 \mathrm{kpc} ; L_{\mathrm{IR}}\left(150^{\circ}\right.$ $\mathrm{K}$ blackbody, ground) $=1.7 \times 10^{37} \mathrm{ergs} \mathrm{s}^{-1}$ $=4.4 \times 10^{3} L_{\odot} ; L_{\mathrm{IR}}\left(155^{\circ} \mathrm{K}\right.$ blackbody, rocket) $=6.2 \times 10^{38} \mathrm{ergs} \mathrm{s}^{-1}=1.6 \times 10^{5} \mathrm{~L}_{\odot}$. 
7635 , immersed in the general $\mathrm{H}$ II region Sharpless $162 . \mathrm{BD}+60^{\circ} 2522$ lies within the brightest portion of the ring. The diameter of the ring is approximately $3^{\prime}$ while the general $\mathrm{H}$ II region extends over some $10^{\circ}$. Consequently the rocket beam would include most of S162 as well as NGC 7635.

Infrared luminosities have been determined from the ground-based and rocket data. The method involves using the color temperature, discussed above, and normalizing a blackbody of this temperature to the observed fluxes, thus yielding the total flux. The luminosity of NGC 7635/S162 as seen by the rocket was derived by assuming that a $180^{\circ} \mathrm{K}$ blackbody was still relevant, and by scaling up the ground-based luminosity by the ratio of the $20-\mu$ rocket flux to the extrapolated $20-\mu$ ground-based flux. All such estimates of luminosity are of necessity lower limits to the true luminosity, due to the unknown nature of the spectrum beyond $20 \mu$. These estimates are appended to table 1. Also tabulated is $L_{\mathrm{UV}}$, the luminosity shortward of the Lyman continuum for NGC 7635/S162. This was estimated by applying the formula relating Lyman continuum luminosity and radio continuum observations (Soifer, Pipher, and Houck 1972 ) to radio flux measurements at four different frequencies, given by Gebel (1968), and taking the average of the derived luminosities. A detailed discussion of the radio data for NGC 7635/S162 is given by Johnson (1972), from which it is apparent that the fluxes given by Gebel refer to the whole $\mathrm{H}$ II region, rather than just the ring of NGC 7635, and are therefore the most appropriate for our determination of Lyman continuum luminosity. The distance to NGC 7635 used in the above estimates of luminosity is the kinematic value of $3.5 \mathrm{kpc}$ determined by Georgelin and Georgelin (1970).

It can be seen from table 1 that the values of $L_{\mathrm{UV}}$ and the rocket-infrared luminosity are comparable. It is interesting to note that agreement occurs at a relatively short wavelength limit in the infrared, whereas such agreement normally results only when far-infrared $(\sim 100 \mu)$ flux is included (Soifer et al. 1972).

$\mathrm{BD}+60^{\circ} 2522$ has been classified as an O6.5 IIIf star by Conti and Alschuler (1971), and such stars are known to exhibit signs of high-velocity mass outflow (Morton, Jenkins, and Brooks 1969). One possible interpretation of all the infrared data for NGC $7635 / \mathrm{S} 162$ is that one is seeing thermal reradiation by dust grains forming in the mass outflow from the Of star. Such a model could explain both the central peak in the infrared emission and its extended nature. We would argue against the dust seen in the vicinity of $\mathrm{BD}+60^{\circ} 2522$ being preexistent, on the grounds that sputtering by energetic stellar-wind particles would rapidly destroy such dust. A more detailed discussion of this model will be given in a forthcoming paper.

\section{b) IC 1470}

IC 1470 (also known as Sharpless 156) is a small red nebula of about 1'.5 diameter, with a faint $\mathrm{O}$ star near its center. Table 2 lists the ultraviolet and infrared luminosities for IC 1470 , derived by the same methods as for NGC 7635. The radio data were taken from Felli and Churchwell (1972), and the distance of $4.3 \mathrm{kpc}$ is again from Georgelin and Georgelin (1970). The rocket infrared luminosity is significantly greater than the value of $L_{\mathrm{Uv}}$, perhaps indicating a dust-limited $\mathrm{H}$ II region.

In a photograph of IC 1470 by Pease (1920), dust lanes are clearly visible across the nebula. The $U B V$ data for the central star also indicate considerable absorption due to dust. In a recent paper, Hoglund and Gordon (1973) report the detection of a formaldehyde absorption feature and an associated $\mathrm{OH}$ emission feature in the Perseus arm, implying the existence of a large dust complex. Their radio map of this complex overlies IC 1470, a fact not recognized by those authors. The coincidence between the radial velocities of $\mathrm{H}_{2} \mathrm{CO}, \mathrm{OH}$, and $\mathrm{H}$ I listed by Hoglund and Gordon, and the interferometric radial velocity of IC 1470 due to Georgelin and Georgelin (1970), implies a common distance. We have mapped the region of IC 1470 in the radio continuum 
at $6 \mathrm{~cm}$ using the 85 -foot $(26-\mathrm{m})$ Hat Creek dish and find a strong emission source, the existence of which complicates the interpretation of the formaldehyde absorption data. Our radio data will be described more fully in a later paper.

It may be possible to interpret the infrared emission from this object as being due to the same mechanism proposed for NGC 7635. The central star of IC 1470 has been classified as an $\mathrm{O} 7$ star in the past, but our estimate of its total luminosity exceeds that of $\mathrm{BD}+60^{\circ} 2522$, suggesting that it might in fact be an Of star. Unfortunately it is too faint to have been so far classified as such, but it would be most useful if a spectrum of sufficient resolution could be taken to settle this question.

However, the apparent large quantities of dust in the region of IC 1470 could permit an alternative explanation in which IC 1470 is a newly formed H II region, just becoming optically visible, and situated in a region of ongoing star formation. The concentration of dust around the central star could then be interpreted as the remnant of a protostellar cocoon not yet fully dispersed.

We thank Dr. N. J. Woolf for observing time at Mount Lemmon, and Drs. R. G. Walker and S. D. Price for a copy of the AFCRL infrared catalog. Support for infrared astronomy at the University of California, Berkeley is under NFC grant GP 31592 X. M. B. acknowledges the receipt of an ESRO-NASA fellowship.

\section{REFERENCES}

Allen, D. A. 1973, M.N.R.A.S., 161, 145.

Cohen, M. 1973, M.N.R.A.S., 161, 85 .

Conti, P. S., and Alschuler, W. R. 1971, Ap. J., 170, 325.

Felli, M., and Churchwell, E. 1972, Astr. and Ap. Suppl., 5, 369.

Gebel, W. L. 1968, Ap. J., 153, 743.

Georgelin, Y. P., and Georgelin, Y. M. 1970, Astr. and Ap., 6, 349.

Hiltner, W. A. 1956, Ap. J. Suppl., 2, 389.

Hoglund, B., and Gordon, M. A. 1973, Ap. J., 182, 45.

Johnson, H. M. 1955, Ap. J., 121, 604.

- 1972, Seventeenth International Astrophysical Symposium held at Liège, p. 345.

Kostjakova, E. B., Saveleva, M. V., Dokuchaeva, O. D., and Noskova, R. I. 1968, IAU Symposium 34 (New York: Springer-Verlag), p. 317.

Morton, D. C., Jenkins, E. B., and Brooks, N. H. 1969, Ap. J., 155, 875.

Pease, F. G. 1920, Ap. J., 51, 276.

Price, S. D., and Walker, R. G. 1973, in preparation.

Roques, P. E. 1973, Griffith Obs., 37, 7.

Soifer, B. T., Pipher, J. L., and Houck, J. R. 1972, Ap. J., 177, 315. 